\title{
Research on the Recovery of Irregular Flights under Uncertain Conditions
}

\author{
Xiangfei Meng, Xiaoyu Lin, Hualei Zhao, Jing Yang \\ Department of Service Supporting, Air Force Command College, Beijing, China \\ Email: mengxiangfeikgd@163.com
}

How to cite this paper: Meng, X.F., Lin, X.Y., Zhao, H.L. and Yang, J. (2021) Research on the Recovery of Irregular Flights under Uncertain Conditions. World Journal of Engineering and Technology, 9, 755764.

https://doi.org/10.4236/wjet.2021.94052

Received: May 28, 2021

Accepted: October 22, 2021

Published: October 25, 2021

\begin{abstract}
This paper mainly studies the problem of irregular flights recovery under uncertain conditions. Based on the analysis of the uncertain factors affecting the flight, taking the total delay time and the total cost of flight delay as the objective function, and considering the constraints of flight plan and passenger journey, an uncertain objective programming model is constructed. Finally, taking OVS airport temporarily closed due to bad weather as an example, the results show that better quality optimization scheme can be obtained by integrating passenger recovery with narrow sense flight recovery stage and implementing integrated recovery.
\end{abstract}

\section{Keywords}

Recovery of Irregular Flights, Uncertainty Theory, Multi-Objective Programming

\section{Introduction}

At present, China's air transport industry is in a period of rapid development, and the market demand is growing. Airlines strive for greater profits by making compact flight plans and reducing costs. However, in the implementation of flight plan, it is often affected by many emergencies and deviates from the original plan, resulting in irregular flights, such as bad weather, major geological disasters, military exercises, accidents, aircraft failures, etc. [1]. When the flight is delayed or cancelled, it is necessary to re-plan and resume the passenger journey according to the passenger destination and existing flight resources, so that the disturbed flights can quickly return to normal. The problem of irregular flight recovery not only has complex objective function and many constraints, but also requires high timeliness of calculation [2]. With the current technical conditions, it is still unable to solve the problem of large-scale irregular flights recov- 
ery in a short time. Therefore, the solution process is usually divided into three sub-problems, namely aircraft path recovery, crew recovery and passenger recovery. Although the phased solution simplifies the problem, it is difficult to get the global optimal solution. Therefore, the current research focuses on the integrated recovery of irregular flights [3]. A series of mathematical models and solutions for the irregular flights integration recovery problem are given in the Literature [4] [5] [6] [7]. Generally speaking, the model is large in scale, high in complexity and difficult to solve. Therefore, the idea of dividing the whole model into one main model and three sub models is proposed, and then iterative solution is proposed. Although the thinking path mentioned in this paper is easy to solve, there is no information interaction among the sub models, and the result of the solution is improved but not ideal. Liu use the time period approximate network model and resource allocation model to solve the integrated recovery problem of irregular flights, calculates the lower bound of the approximate optimal solution and the optimal solution, estimates the gap between the two. Then, taking the United States continuous airlines as an example, the availability of the method is illustrated, The results show that the method proposed in this paper is better than random search method in solving the problem, but the feasibility of the method in practical problems cannot be explained because of the small amount of data.

There are many reasons for irregular flights, some of which cannot be accurately described by numbers or models. For example, if the flight is delayed due to severe weather such as thunderstorms, the flight cannot be recovered until the weather improves. The period from the start of the delay to the recovery of the flight is difficult to accurately predict even based on the information of the weather forecast. When the weather reaches take-off or landing conditions, the air traffic control department may control the air traffic flow, causing the aircraft to stay at the airport unable to perform flight tasks. Flight delays caused by many reasons are more difficult to recover. Dispatchers can only rely on Make appropriate adjustments to experience. As flight delays or cancellations seriously affect the passengers' itinerary, passengers will be disappointed with the airline's services and capabilities, and the airline's reputation will decline, which will cause potential economic losses to the airline. In modeling, it is difficult to use one or a set of accurate figures to measure the degree of passenger disappointment to the airline and the airline's potential economic loss [8] [9] [10]. Therefore, it is described by introducing uncertain variables. It can be seen that there are many factors that cause flight irregularities. Under the combined effect of these factors, the uncertainty of flight recovery problems is mainly manifested in the impact of flight delays or cancellations on passengers and flight delays or cancellation costs. Therefore, the problem of irregular flights recovery is a typical uncertain multi-objective planning problem.

\section{Modeling of Irregular flights Recovery under Uncertain Conditions}

The irregular flights recovery considering aircraft path and passenger itinerary 
integrates aircraft path recovery and passenger recovery, and abstracts the objectives and constraints involved in these two recovery problems as objective functions and constraints. The meanings of the parameters involved in the model are shown in Table 1.

\subsection{Objective Function Analysis}

The objective function of the irregular flights recovery problem includes the total time of passenger delay and the total cost of flight delay.

- Total time of passengers delay. According to the relevant regulations of the Civil Aviation Administration, the total time of passengers delay refers to the product of the flight delay time and the number of passengers, as shown in Equation (1).

$$
T_{a}^{f}\left(t_{a}^{f}(k), \xi\right)= \begin{cases}\xi_{1} \cdot\left(N_{E}(f)+N_{B}(f)\right) \cdot t_{a}^{f}(k) \cdot k, & 0 \leq k<24 \\ \xi_{2} \cdot\left(N_{E}(f)+N_{B}(f)\right) \cdot t_{a}^{f}(k) \cdot k, & 24 \leq k<60 . \\ \xi_{3} \cdot\left(N_{E}(f)+N_{B}(f)\right) \cdot t_{a}^{f}(k) \cdot k, & 60 \leq k \leq 96\end{cases}
$$

where $T_{a}^{f}$ is the total delay time for flight a to execute flight $f, \xi_{1}, \xi_{2}, \xi_{3}$ are the uncertainty weight factors following the distributions $\mathcal{L}(0,0.3), \mathcal{L}(0.3,0.7)$ and $\mathcal{L}(0.7,1.0)$, respectively, $k$ represents the $k$-th time unit, with 5 minutes as one time unit.

According to the relevant regulations of the Civil Aviation Administration, the flight cancellation time can be converted into a delay of 8 hours. Therefore, the total time of passenger delay is

$$
T_{F}=\sum_{f \in F} \sum_{a \in A} x_{a}^{f} \cdot T_{a}^{f}\left(t_{a}^{f}(k), \xi\right)+\sum_{f \in F} 96\left(N_{E}(f)+N_{B}(f)\right) \cdot y_{f} .
$$

- The total cost of flights delay. The total cost of flight delay includes direct cost and indirect cost. Direct costs include the cost of airline compensation and aircraft in the air and ground holding and canceled flights.

The cost of airline compensation. The Civil Aviation Administration's compensation standard for flight delays stipulates: if the flight is delayed due to the airline's own reasons, the delay is between 4 hours and 8 hours, the passenger will be compensated not less than $200 ¥$; the flight will be cancelled if the delay exceeds 8 hours, and each passenger will be compensated Not less than $400 ¥$. On the basis of the compensation standard, the airline makes compensation in combination with the actual delay. The passenger compensation cost can be calculated by Equation (3).

$$
C P_{a}^{f}(\boldsymbol{\eta}, k)=\left\{\begin{array}{cl}
0, & 0 \leq k<48 \\
\eta_{1} \cdot\left(q_{f}^{b}+q_{f}^{e}\right), & 48 \leq k<96 . \\
\eta_{2} \cdot\left(q_{f}^{b}+q_{f}^{e}\right), & k \geq 96
\end{array}\right.
$$

where $C P_{a}^{f}$ is the cost of compensation to passengers due to the delay of flight $f$ implemented by aircraft $a ; \eta_{1}, \eta_{2}$ are compensation amount for each passenger under different circumstances following the distributions 
Table 1. The meanings of the parameters involved in the model.

\begin{tabular}{|c|c|c|c|}
\hline Parameters & Meanings & Parameters & Meanings \\
\hline$f$ & Flights & $f_{V}$ & VIP flights \\
\hline$f_{m}$ & Important flights & $f_{g}$ & Ordinary flights \\
\hline$A$ & The set of available aircrafts & $a$ & Aircrafts \\
\hline$L$ & The set of type of aircrafts & 1 & The type of aircrafts \\
\hline$p$ & Airports & $P$ & The set of Airports \\
\hline$F$ & The set of flights & $F_{V}$ & The set of disturbed VIP flights \\
\hline$F_{m}$ & The set of disturbed important flights & $F_{g}$ & The set of disturbed regular flights \\
\hline$y_{f}$ & $\begin{array}{l}\text { If flight } f \text { is cancelled, the value is } 1 \text {, otherwise it is } \\
0\end{array}$ & $x_{a}^{f}$ & $\begin{array}{l}\text { If airplane a executes flight } f \text {, the value is } 1 \text {, other- } \\
\text { wise it is } 0\end{array}$ \\
\hline$t_{a}^{f}(k)$ & $\begin{array}{l}\text { If the plane a executes the flight } f \text {, it takes off at } \\
\text { the time unit } k \text { of the delay, then the value is } 1 \text {, } \\
\text { otherwise it is } 0\end{array}$ & $N_{p}^{G A T E}\left(t_{1}, t_{2}\right)$ & $\begin{array}{l}\text { The number of gates available at airport } p \text { during } \\
\left(t_{1}, t_{2}\right), \quad p \in P\end{array}$ \\
\hline$N_{p}^{\text {AFTER }}(l)$ & $\begin{array}{l}\text { The number of aircrafts of model } l \text { which required } \\
\text { to be parked at airport } p \text { after the restoration }\end{array}$ & $N_{B}(l)$ & $\begin{array}{l}\text { The number of business class seats of the aircraft } \\
\text { belongs to type } l\end{array}$ \\
\hline$N_{B}(f)$ & $\begin{array}{l}\text { The number of business class passengers on flight } \\
f\end{array}$ & $N_{E}(l)$ & $\begin{array}{l}\text { The number of economy class seats of the aircraft } \\
\text { belongs to type } 1\end{array}$ \\
\hline$N_{E}(f)$ & $\begin{array}{l}\text { The number of economy class passengers on flight } \\
f\end{array}$ & $p_{f}^{B}$ & The fare for business class seats on flight $f$ \\
\hline$p_{f}^{E}$ & The fare for economy class seats on flight $f$ & $N(l)$ & $\begin{array}{l}\text { The total number of seats of the aircraft belongs to } \\
\text { type } 1\end{array}$ \\
\hline$p g$ & The itinerary of the disturbed passenger & $I(p, t)$ & $\begin{array}{l}\text { The set of inbound flights parking at airport } p \text { at } \\
\text { moment } t\end{array}$ \\
\hline$P G$ & $\begin{array}{l}\text { The set of the itinerary route of the disturbed pas- } \\
\text { senger (departure airport to arrival airport) }\end{array}$ & $O(p, t)$ & $\begin{array}{l}\text { The set of outbound flights parking at airport } p \text { at } \\
\text { moment } t\end{array}$ \\
\hline $\bar{F}(l)$ & $\begin{array}{l}\text { The flight set of aircraft type } I \text { cannot be allocated, } \\
l \in L, \bar{F}(l) \in F\end{array}$ & $\bar{P}\left(t_{1}, t_{2}\right)$ & $\begin{array}{l}\text { The set of airports closed due to inclement weather } \\
\text { during }\left(t_{1}, t_{2}\right)\end{array}$ \\
\hline$R(p g)$ & $\begin{array}{l}\text { The set of alternative itineraries for disturbed } \\
\text { passengers, } \quad p g \in P G\end{array}$ & $b_{p, f}^{L A S T}$ & $\begin{array}{l}\text { On the same day, flight } f \text { finally lands at airport } p \text {, } \\
\text { it is } 1 \text {, otherwise it is } 0\end{array}$ \\
\hline$b_{p, f}^{F L T}$ & $\begin{array}{l}\text { If the flight } f \text { stops at the airport } p \text { within the spe- } \\
\text { cified time, the value is } 1 \text {, otherwise it is } 0\end{array}$ & $b_{f, r}^{P A X}$ & $\begin{array}{l}\text { If the flight } f \text { is on route } r \text { of the disturbed passen- } \\
\text { gers, the value is } 1 \text {, otherwise it is } 0, r \in R(p g)\end{array}$ \\
\hline$b_{a, l}^{F L T}$ & $\begin{array}{l}\text { If aircraft a belongs to type } l \text {, it is } 1 \text {, otherwise it is } \\
0\end{array}$ & $n_{r}^{P A X}$ & $\begin{array}{l}\text { The number of passengers on flight } f \text { on route } r \\
r \in R(p g)\end{array}$ \\
\hline$x_{l}^{f}$ & $\begin{array}{l}\text { The aircraft belongs to type } l \text { is assigned to flight } f \text {, } \\
\text { then it is } 1 \text {, otherwise it is } 0 \quad l \in L, f \in F\end{array}$ & $Z_{r}^{p g}$ & $\begin{array}{l}\text { The number of passengers whose flight route is on } \\
r \text { and the departure and arrival airport is } p g \\
r \in R(p g), p g \in P G\end{array}$ \\
\hline
\end{tabular}

$\mathcal{L}(200,300), \mathcal{L}(400,600)$.

The cost of aircraft air/ground holding. The recovery of irregular flights is mainly to study how to reasonably reschedule the flight plan. Therefore, the cost 
is mainly the cost of aircraft ground waiting, including the cost of aircraft parking at the airport and the cost of aircraft depreciation, which can be calculated by Equation (4).

$$
C W_{a}^{f}=(\beta+v) \cdot t_{a}^{f}(k) \cdot k .
$$

where $C W_{a}^{f}$ is the ground holding cost of the delay or cancellation of flight $f$ implemented by aircraft $a ; \beta$ is the fee charged for every minute of aircraft $a$ stopping at the airport; $v$ is the depreciation cost per minute of aircraft $a$.

The cost of cancelling flights. It is calculated as in Equation (5).

$$
C C_{a}^{f}=p_{a}^{f} \cdot y_{f}
$$

where $C C_{a}^{f}$ is the cost incurred by aircraft a due to the cancellation of flight $f$.

Indirect costs refer to the damage caused by the airline's reputation due to irregular flights, and passengers no longer choose their flights in future trips,, as shown in Equation (6).

$$
C I_{a}^{f}(\xi, k)= \begin{cases}\left(p_{f}^{B} \cdot N_{B}(f)+p_{f}^{E} \cdot N_{E}(f)\right) \cdot \xi_{1}, & 0 \leq k<24 \\ \left(p_{f}^{B} \cdot N_{B}(f)+p_{f}^{E} \cdot N_{E}(f)\right) \cdot \xi_{2}, & 24 \leq k<60 . \\ \left(p_{f}^{B} \cdot N_{B}(f)+p_{f}^{E} \cdot N_{E}(f)\right) \cdot \xi_{3}, & 60 \leq k \leq 96\end{cases}
$$

where $C I_{a}^{f}$ is the indirect cost of aircraft a due to the cancellation or delay of flight $f$.

Therefore, the total cost of flights delay can be calculated by Equation (7).

$$
C_{F}=\sum_{f \in F} \sum_{a \in A} x_{a}^{f} \cdot\left[C P_{a}^{f}(\boldsymbol{\eta})+C W_{a}^{f}+C C_{a}^{f}+C I_{a}^{f}(\xi, k)\right] .
$$

\subsection{Constraints Analysis}

- Flights schedule constraints.

Flights coverage constraint. Each flight is executed by one aircraft, or the flight is cancelled. The constraint is described as Equation (8).

$$
\sum_{a \in A}\left(x_{a}^{f}+y_{f}\right)=1, \quad \forall f \in F .
$$

Aircraft flow balance constraint. After the completion of the recovery, the number and types of aircraft parked at each airport meet the requirements of the plan. The constraint is described as Equation (9).

$$
\sum_{f \in F} b_{p, f}^{\text {LAST }} \cdot x_{l}^{f} \geq N_{p}^{\text {AFTER }}(l), \quad \forall p \in P, l \in L .
$$

Parking space constraint. The airport must have enough parking spaces to ensure that flights take off and land normally. The constraint is described as Equation (10)

$$
\sum_{a \in A}\left(\sum_{f \in I(p, t)} x_{a}^{f}-\sum_{f \in O(p, t)} x_{a}^{f}\right) \leq N_{p}^{G A T E}\left(t_{1}, t_{2}\right), \quad \forall p \in P .
$$

VIP flights important precedence constraint. Minimize delays on important flights. The constraint is described as Equation (11). 


$$
x_{a}^{f_{v}} \geq x_{a}^{f_{m}} \geq x_{a}^{f_{g}}, \forall a \in A, f_{v} \in F_{v}, f_{m} \in F_{m}, f_{g} \in F_{g} .
$$

Airport closure constraint. The airport was closed due to bad weather and the aircraft could not be taken off or landed during this period. The constraint is described as Equation (12).

$$
\sum_{f \in F} \sum_{a \in A} b_{p, f}^{F L T} \cdot x_{a}^{f}=0, \quad \forall p \in \bar{P}\left(t_{1}, t_{2}\right) .
$$

Aircraft type matching constraint. Different aircraft types are not allowed to be exchanged. The constraint is described as Equation (13).

$$
\sum_{f \in \bar{F}(l)} x_{l}^{f}=0, \quad \forall l \in L .
$$

Airplane seats constraint. The seats of the aircraft allocated to a certain flight should meet the needs of passengers. The constraint is described as Equation (14).

$\sum_{l \in L} \sum_{a \in A} b_{a, l}^{F L T} \cdot x_{a}^{f} \cdot N_{B}(l) \geq N_{B}(f), \forall f \in F, \sum_{l \in L} \sum_{a \in A} b_{a, l}^{F L T} \cdot x_{a}^{f} \cdot N_{E}(l) \geq N_{E}(f), \forall f \in F$.

- Passengers constraints.

Passenger number constraints. Ensure that the new flight plan will not cause overbooking. The constraint is described as Equation (15).

$$
\sum_{p g \in P G} \sum_{r \in R(p g)} b_{f, r}^{P A X} \cdot z_{r}^{p g} \leq \sum_{a \in A} \sum_{l \in L} x_{a}^{f} \cdot b_{a, l}^{F L T} \cdot N(l), \quad \forall f \in F .
$$

Passengers itinerary constraints. Ensure that the passenger itinerary in the new plan is consistent with the original plan. The constraint is described as Equation (16).

$$
\sum_{r \in R(p g)} Z_{r}^{p g}=n_{r}^{P A X}, \quad \forall p g \in P G
$$

- Decision variable integer constraints.

$$
\begin{cases}x_{a}^{f} \in\{0,1\}, & \forall f \in F, a \in A \\ y_{f} \in\{0,1\}, & \forall f \in F \\ x_{l}^{f} \in\{0,1\}, & \forall l \in L, f \in F \\ z_{r}^{p g} \in Z^{+}, & \forall r \in R(p g), p g \in P G\end{cases}
$$

Therefore, the uncertain multi-objective planning model for the recovery of irregular flights is shown as Equation (18).

$$
\left\{\begin{array}{l}
\min T_{F}=\sum_{f \in F} \sum_{a \in A} x_{a}^{f} \cdot T_{a}^{f}\left(t_{a}^{f}(k), \xi\right)+\sum_{f \in F} 96\left(N_{E}(f)+N_{B}(f)\right) \cdot y_{f} \\
\min C_{F}=\sum_{f \in F} \sum_{a \in A} x_{a}^{f} \cdot\left[C P_{a}^{f}(\boldsymbol{\eta})+C W_{a}^{f}+C C_{a}^{f}+C I_{a}^{f}(\xi, k)\right] \\
\text { s.t. } \\
\sum_{a \in A}\left(x_{a}^{f}+y_{f}\right)=1, \quad \forall f \in F ; \sum_{f \in F} b_{p, f}^{L A S T} \cdot x_{l}^{f} \geq N_{p}^{A F T E R}(l), \quad \forall p \in P, l \in L ; \\
\sum_{a \in A}\left(\sum_{f \in I(p, t)} x_{a}^{f}-\sum_{f \in O(p, t)} x_{a}^{f}\right) \leq N_{p}^{G A T E}\left(t_{1}, t_{2}\right), \quad \forall p \in P ; \\
x_{a}^{f_{v}} \geq x_{a}^{f_{m}} \geq x_{a}^{f_{g}}, \quad \forall a \in A, f_{v} \in F_{v}, f_{m} \in F_{m}, f_{g} \in F_{g} ;
\end{array}\right.
$$




$$
\left\{\begin{array}{l}
\sum_{f \in F} \sum_{a \in A} b_{p, f}^{F L T} \cdot x_{a}^{f}=0, \quad \forall p \in \bar{P}\left(t_{1}, t_{2}\right) ; \sum_{f \in \bar{F}(l)} x_{l}^{f}=0, \quad \forall l \in L ; \\
\sum_{l \in L} \sum_{a \in A} b_{a, l}^{F L T} \cdot x_{a}^{f} \cdot N_{B}(l) \geq N_{B}(f), \forall f \in F ; \sum_{l \in L} \sum_{a \in A} b_{a, l}^{F L T} \cdot x_{a}^{f} \cdot N_{E}(l) \geq N_{E}(f), \forall f \in F ; \\
\sum_{p g \in P G} \sum_{r \in R(p g)} b_{f, r}^{P A X} \cdot z_{r}^{p g} \leq \sum_{a \in A} \sum_{l \in L} x_{a}^{f} \cdot b_{a, l}^{F L T} \cdot N(l), \forall f \in F ; \sum_{r \in R(p g)} z_{r}^{p g}=n_{r}^{P A X}, \forall p g \in P G ; \\
x_{a}^{f} \in\{0,1\}, \forall f \in F, a \in A ; y_{f} \in\{0,1\}, \forall f \in F ; t_{a}^{f} \in\{0,1\}, k \in N, \forall a \in A, f \in F ; \\
x_{l}^{f} \in\{0,1\}, \forall l \in L, f \in F ; z_{r}^{p g} \in Z^{+}, \forall r \in R(p g), p g \in P G .
\end{array}\right.
$$

\section{Case Analysis}

Based on the data from the 2017 graduate mathematical modeling contest, we try to solve the problem of irregular flights recovery under uncertain conditions. Due to bad weather, the airport OVS will be closed from 18:00 to 21:00 on May 13, 2016. During this time period, the airport OVS cannot take off and land any aircraft. After this time period (excluding the two times of 18:00 and 21:00), the airport will resume immediately. Due to the limitation of the runway capacity of the OVS airport, no more than 5 planes can take off every 5 minutes, and no more than 5 planes can land. Other airports do not consider runway restrictions.

To simplify the calculation, set May 13, 2016 00:00-00:05 as the first time decision unit, and then every 5 minutes as a time decision unit. For example, 21:00 to 21:05 (excluding 21:05 time) is the 252nd time decision unit, and all flights taking off and landing within this unit time are counted as 21:00.

According to the uncertain multi-objective programming solution method in the Literature [11] [12] [13], the uncertain multi-objective programming model is transformed into a certain single-objective programming model, as shown in Equation (19).

$$
\left\{\begin{array}{l}
\min E\left[\lambda_{1} T_{F}+\lambda_{2} C_{F}\right]+\sigma\left[\lambda_{1} T_{F}+\lambda_{2} C_{F}\right] \\
\text { s.t. } \\
\sum_{a \in A}\left(x_{a}^{f}+y_{f}\right)=1, \quad \forall f \in F ; \sum_{f \in F} b_{p, f}^{L A S T} \cdot x_{l}^{f} \geq N_{p}^{A F T E R}(l), \quad \forall p \in P, l \in L ; \\
\sum_{a \in A}\left(\sum_{f \in I(p, t)} x_{a}^{f}-\sum_{f \in O(p, t)} x_{a}^{f}\right) \leq N_{p}^{G A T E}\left(t_{1}, t_{2}\right), \quad \forall p \in P ; \\
x_{a}^{f_{v}} \geq x_{a}^{f_{m}} \geq x_{a}^{f_{g}}, \forall a \in A, f_{v} \in F_{v}, f_{m} \in F_{m}, f_{g} \in F_{g} ; \\
\sum_{f \in F} \sum_{a \in A} b_{p, f}^{F L T} \cdot x_{a}^{f}=0, \quad \forall p \in \bar{P}\left(t_{1}, t_{2}\right) ; \sum_{f \in \bar{F}(l)} x_{l}^{f}=0, \quad \forall l \in L ; \\
\sum_{l \in L} \sum_{a \in A} b_{a, l}^{F L T} \cdot x_{a}^{f} \cdot N_{B}(l) \geq N_{B}(f), \forall f \in F ; \sum_{l \in L} \sum_{a \in A} b_{a, l}^{F L T} \cdot x_{a}^{f} \cdot N_{E}(l) \geq N_{E}(f), \forall f \in F ; \\
\sum_{p g \in P G} \sum_{r \in R(p g)} b_{f, r}^{P A X} \cdot z_{r}^{p g} \leq \sum_{a \in A} \sum_{l \in L} x_{a}^{f} \cdot b_{a, l}^{F L T} \cdot N(l), \forall f \in F ; \sum_{r \in R(p g)} z_{r}^{p g}=n_{r}^{P A X}, \forall p g \in P G ; \\
x_{a}^{f} \in\{0,1\}, \forall f \in F, a \in A ; y_{f} \in\{0,1\}, \forall f \in F ; t_{a}^{f} \in\{0,1\}, k \in N, \forall a \in A, f \in F ; \\
x_{l}^{f} \in\{0,1\}, \forall l \in L, f \in F ; z_{r}^{p g} \in Z^{+}, \forall r \in R(p g), p g \in P G .
\end{array}\right.
$$

where 


$$
\begin{aligned}
& E\left[\lambda_{1} T_{F}+\lambda_{2} C_{F}\right]=\lambda_{1}\left\{\sum_{f \in F} \sum_{a \in A} x_{a}^{f} \cdot E\left[T_{a}^{f}\left(t_{a}^{f}(k), \xi\right)\right]+\sum_{f \in F} 96\left(N_{E}(f)+N_{B}(f)\right) \cdot y_{f}\right\} \\
& +\lambda_{2} \sum_{f \in F} \sum_{a \in A} x_{a}^{f}\left\{E\left[C P_{a}^{f}(\boldsymbol{\eta})\right]+C W_{a}^{f}+C C_{a}^{f}+E\left[C I_{a}^{f}(\xi, k)\right]\right\}, \\
& E\left[T_{a}^{f}\left(t_{a}^{f}(k), \xi\right)\right]= \begin{cases}0.15\left(N_{E}(f)+N_{B}(f)\right) \cdot t_{a}^{f}(k) \cdot k, & 0 \leq k<24 \\
0.5\left(N_{E}(f)+N_{B}(f)\right) \cdot t_{a}^{f}(k) \cdot k, & 24 \leq k<60, \\
0.85\left(N_{E}(f)+N_{B}(f)\right) \cdot t_{a}^{f}(k) \cdot k, & 60 \leq k \leq 96\end{cases} \\
& E\left[C P_{a}^{f}(\boldsymbol{\eta}, k)\right]=\left\{\begin{array}{cl}
0, & 0 \leq k<48 \\
250\left(N_{E}(f)+N_{B}(f)\right), & 48 \leq k<96, \\
500\left(N_{E}(f)+N_{B}(f)\right), & k \geq 96
\end{array}\right. \\
& E\left[C I_{a}^{f}(\xi, k)\right]= \begin{cases}0.15\left(p_{f}^{B} \cdot N_{B}(f)+p_{f}^{E} \cdot N_{E}(f)\right), & 0 \leq k<24 \\
0.5\left(p_{f}^{B} \cdot N_{B}(f)+p_{f}^{E} \cdot N_{E}(f)\right), & 24 \leq k<60, \\
0.85\left(p_{f}^{B} \cdot N_{B}(f)+p_{f}^{E} \cdot N_{E}(f)\right), & 60 \leq k \leq 96\end{cases} \\
& \left.\sigma\left[\lambda_{1} T_{F}+\lambda_{2} C_{F}\right]=\sqrt{V\left[\lambda_{1} T_{F}+\lambda_{2} C_{F}\right.}\right] \\
& =\sqrt{\sum_{f \in F} \sum_{a \in A} V\left[\left(\lambda_{1} x_{a}^{f} \cdot T_{a}^{f}\left(t_{a}^{f}(k), \xi\right)+\lambda_{2} x_{a}^{f} \cdot C I_{a}^{f}(\xi, k)\right)\right]+\lambda_{2}^{2}\left(x_{a}^{f}\right)^{2} \sum_{f \in F} \sum_{a \in A} V\left[C P_{a}^{f}(\boldsymbol{\eta}, k)\right]} . \\
& V\left[\lambda_{1} x_{a}^{f} \cdot T_{a}^{f}\left(t_{a}^{f}(k), \xi\right)+\lambda_{2} x_{a}^{f} \cdot C I_{a}^{f}(\xi, k)\right] \\
& = \begin{cases}3\left[\lambda_{1} x_{a}^{f} \cdot n_{a}^{f} \cdot t_{a}^{f}(k) \cdot k+\lambda_{2} x_{a}^{f} \cdot\left(p_{f}^{B} \cdot N_{B}(f)+p_{f}^{E} \cdot N_{E}(f)\right)\right]^{2} / 400, & 0 \leq k<24 \\
{\left[\lambda_{1} x_{a}^{f} \cdot n_{a}^{f} \cdot t_{a}^{f}(k) \cdot k+\lambda_{2} x_{a}^{f} \cdot\left(p_{f}^{B} \cdot N_{B}(f)+p_{f}^{E} \cdot N_{E}(f)\right)\right]^{2} / 30,} & 24 \leq k<60, \\
17\left[\lambda_{1} x_{a}^{f} \cdot n_{a}^{f} \cdot t_{a}^{f}(k) \cdot k+\lambda_{2} x_{a}^{f} \cdot\left(p_{f}^{B} \cdot N_{B}(f)+p_{f}^{E} \cdot N_{E}(f)\right)\right]^{2} / 40, & 60 \leq k \leq 96\end{cases} \\
& V\left[C P_{a}^{f}(\boldsymbol{\eta}, k)\right]=\left\{\begin{array}{cl}
0, & 0 \leq k<48 \\
12500\left(N_{B}(f)+N_{E}(f)\right)^{2} / 3, & 48 \leq k<96 . \\
50000\left(N_{B}(f)+N_{E}(f)\right)^{2} / 3, & k \geq 96
\end{array}\right.
\end{aligned}
$$

The inverse binary learning fireworks algorithm is used to solve the model (19).

Figure 1 shows the length of each flight delay in the new flight plan. Similarly, the length of the vertical line indicates the duration of the flight delay. The longer the vertical line, the longer the delay time of the corresponding flight, and vice versa, the shorter; the "*" point indicates that the corresponding flight is not delayed.

It can be seen from Figure 1 and Figure 2 that 116 of the 172 flights were delayed and no flights were cancelled. The total delay was 2360 time units, or 11,800 minutes, and the total delay of passengers was 207,606 time units, or $1,038,030$ minutes; the passengers were required to be compensated for flights delayed for more than 4 hours. 0 , the total delay cost is 1,976,900 ¥. If no measures are taken for irregular flights, that is, all affected flights are postponed for 3 hours, the flight will be delayed for a total of 20,880 minutes, and passengers will be delayed for 1,593,180 minutes, and the total cost of delay will be 3,435,600 $¥$. Considering passenger constraints, the newly formulated flight plan reduces flight delays by 9080 minutes, passenger delays by 555,150 minutes, and delay 


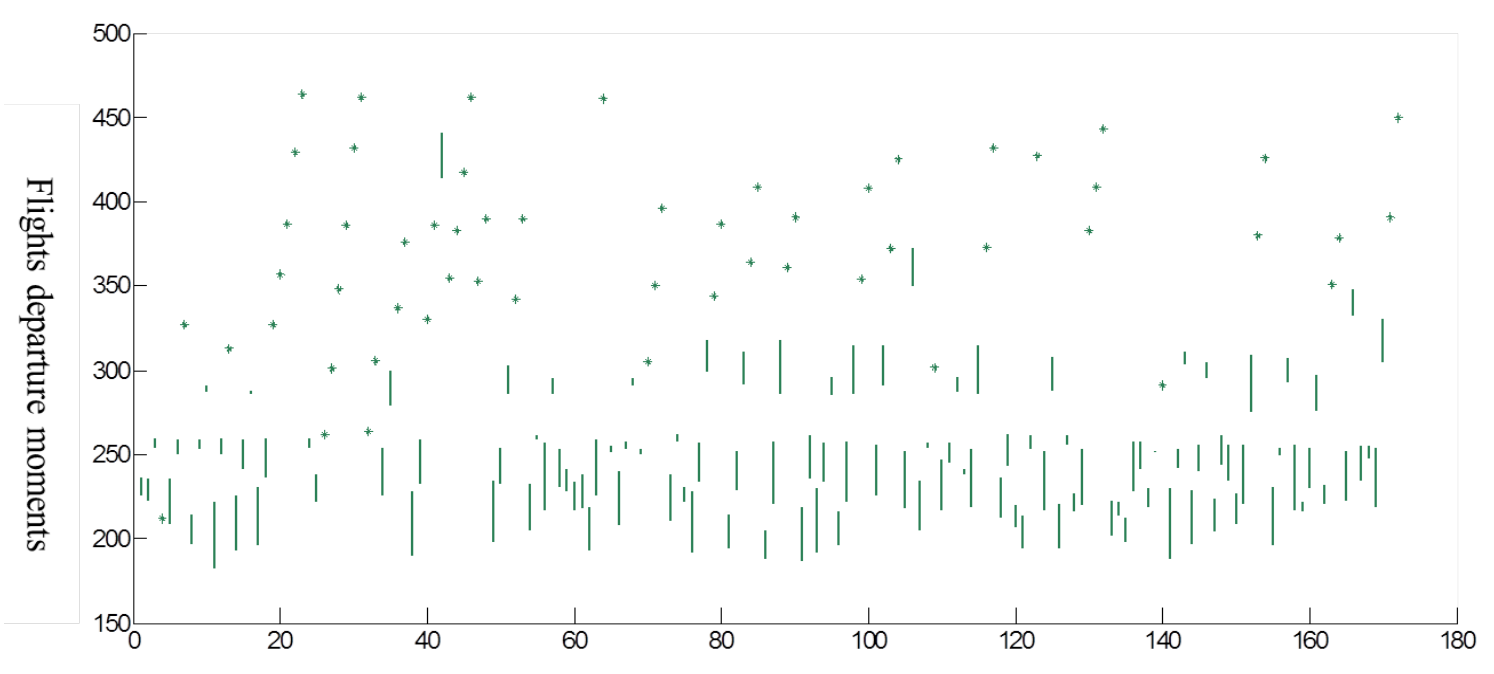

Flights

Figure 1. Statistics of the delay time of new flight plans under uncertain conditions.

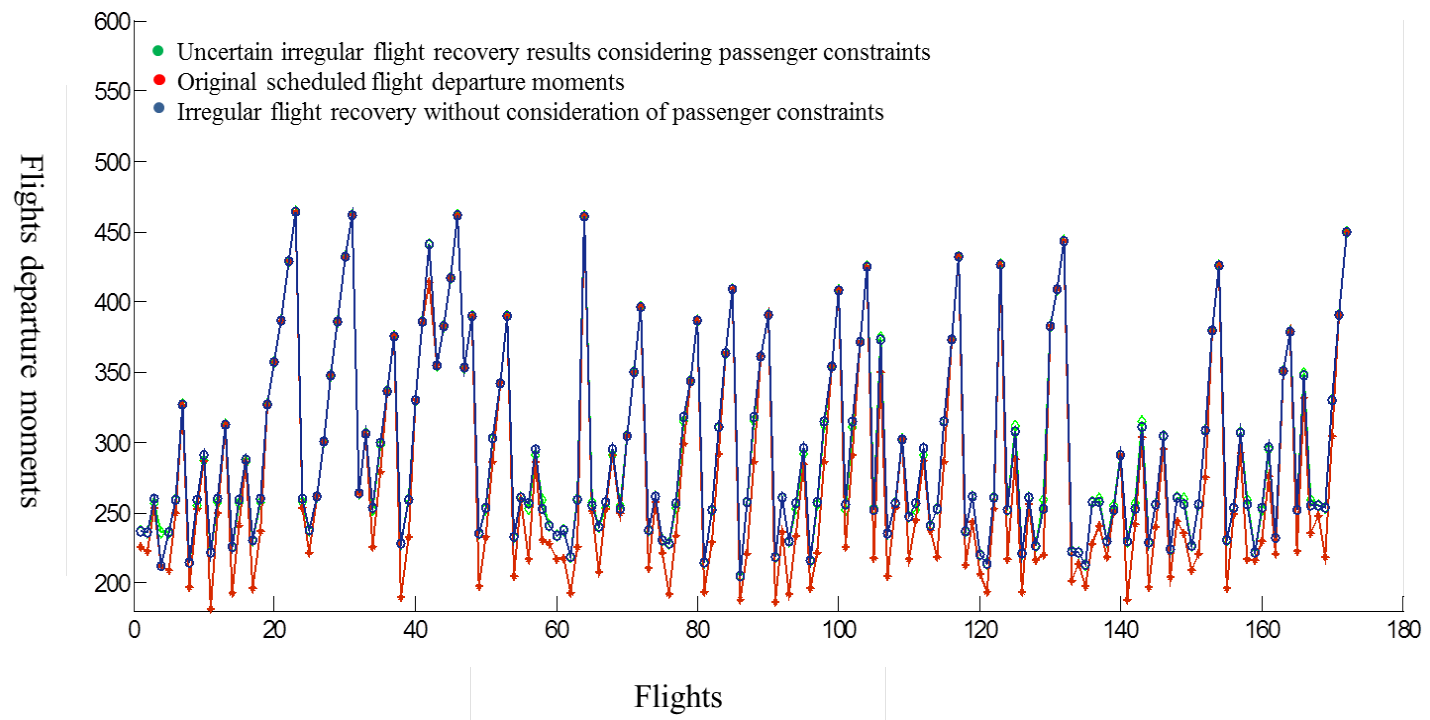

Figure 2. Comparison of the departure time of original flights schedule and the flights after resumption.

costs by $1,458,700 ¥$, which shows the effectiveness of the newly formulated flight plan.

\section{Conclusion}

Based on the analysis of the uncertain factors and basic models that affect the recovery of abnormal flights, this paper constructs an uncertain flight recovery model, and uses the binary learning firework algorithm to solve the problem, which provides a solution for abnormal flight recovery under uncertain conditions. An example for demonstration and verification was proposed, the results show that integrating the two-stage flight recovery into one model for research can obtain a better quality solution. The next step will be to study the integrated 
restoration of irregular flights under uncertain conditions.

\section{Acknowledgements}

This work was supported by National Social Science Foundation of China (No. 2020-SKJJ-C-033) and Natural Science Foundation of Shaanxi Province (No. 2021JQ-368).

\section{Conflicts of Interest}

The authors declare no conflicts of interest regarding the publication of this paper.

\section{References}

[1] Jamili, A. (2017) A Robust Mathematical Model and Heuristic Algorithms for Integrated Aircraft Routing and Scheduling, with Consideration of Fleet Assignment Problem. Journal of Air Transport Management, 58, 21-30.

[2] Zhu, B., Clarke, J.P. and Zhu, J. (2016) Real-Time Integrated Flight Schedule Recovery Problem Using Sampling-Based Approach. Journal of Computational \& Theoretical Nanoscience, 13, 1458-1467.

[3] Thengvall, B.G., Yu, G. and Bard, J.F. (2001) Multiple Fleet Aircraft Schedule Recovery Following Hub Closures. Transportation Research Part a, 35, 289-308.

[4] Maher, S.J. (2015) A Novel Passenger Recovery Approach for the Integrated Airline Recovery Problem. Computers \& Operations Research, 57, 123-137.

[5] Arikan, U., Gurel, S. and Akturk, M.S. (2016) Integrated Aircraft and Passenger Recovery with Cruise Time Controllability. Annals of Operations Research, 236, 1-23.

[6] Bratu, S. and Barnhart, C. (2006) Flight Operations Recovery: New Approaches Considering Passenger Recovery. Journal of Scheduling, 9, 279-298.

[7] Sinclair, K., Cordeau, J.F. and Laporte, G. (2014) Improvements to a Large Neighborhood Search Heuristic for an Integrated Aircraft and Passenger Recovery Problem . European Journal of Operational Research, 233, 234-245.

[8] Li, R. and Liu. G. (2014) An Uncertain Goal Programming Model for Machine Scheduling Problem. Journal of Intelligent Manufacturing, 28, 1-6.

[9] Liu, L., Zhang, B. and Ma, W. (2017) Uncertain Programming Models for Fixed Charge Multi-Item Solid Transportation Problem. Soft Computing, 1, 1-9.

[10] Dalman, H. (2016) Uncertain Programming Model for Multi-Item Solid Transportation Problem. International Journal of Machine Learning \& Cybernetics, 9, 1-9.

[11] Liu, B.D. (2016) Uncertainty Theory. 5th edition, Springer-Verlag, Berlin.

[12] Wang, Z.T., Guo, J.S. and Zheng, M.F. (2014) A New Approach for Uncertain Multiobjective Programming Problem Based on PE Principle. Journal of Industrial and Management Optimization, 11, 13-26.

[13] Meng, X.F., Wang, Y., Li, C., Wang, XY. and Lv, M.L. (2018) Approach for Uncertain Multi-Objective Programming Problems with Correlated Objective Functions under CEV Criterion. Journal of Systems Engineering and Electronics, 29, 1197 1208 . 